\title{
Análisis de la restricción externa de la economía de Cuba en el actual contexto de incertidumbre
}

\author{
Juan Carlos Palacios Cívico
}

\section{Resumen}

La presente investigación se plantea con el doble propósito de contrastar la restricción externa de la economía cubana y de identificar las principales oportunidades y amenazas que esta enfrenta en el actual contexto de incertidumbre. Para ello, se estima un modelo de crecimiento restringido por la balanza de pagos en el que, por primera vez, se hace una estimación del efecto de los diferentes flujos de divisas en el crecimiento de corto y largo plazo de la economía caribeña.

\section{Palabras clave}

Condiciones económicas, crecimiento económico, comercio exterior, balanza de pagos, política económica, modelos econométricos, indicadores económicos, Cuba

\section{Clasificación JEL}

P27, N16, F43

Autor

Juan Carlos Palacios Cívico es Profesor en el Departamento de Historia Económica, Instituciones, Economía Mundial y Política de la Universidad de Barcelona (España). Correo electrónico: jcpalacios@ub.edu. 


\section{Introducción}

La historia reciente de Cuba muestra la imagen de una economía altamente vulnerable a los cambios ocurridos en su entorno. Acontecimientos como la inserción en el Consejo de Ayuda Mutua Económica (CAME), la desaparición de la Unión de Repúblicas Socialistas Soviéticas (URSS) o la integración a la Alianza Bolivariana para los Pueblos de Nuestra América (ALBA) han de ser necesariamente considerados en el análisis del crecimiento económico del país caribeño en las últimas décadas. De igual modo, ese contexto de dependencia externa ayuda a entender la relevancia que pueden tener determinados choques externos, como la crisis política y económica por la que atraviesa la República Bolivariana de Venezuela o el nuevo marco de relaciones con los Estados Unidos, en la determinación de las condiciones materiales de la población cubana durante los próximos años.

Con la presente investigación se persigue, por una parte, contrastar empíricamente la restricción externa que podría estar limitando el crecimiento económico cubano, y, por la otra, estimar de forma desagregada el efecto que pueden tener los principales flujos de divisas (exportaciones, inversión extranjera directa (IED), remesas y créditos con el exterior) en el crecimiento del PIB cubano, con el objeto último de identificar las principales oportunidades y amenazas que se presentan en la actual coyuntura. La metodología propuesta se basa en la estimación econométrica de un modelo de crecimiento restringido por la balanza de pagos y, más concretamente, en el modelo teórico descrito en Thirlwall y Hussain (1982), en el que la renta de un país se expresa en función del crecimiento de las exportaciones, los términos de intercambio y el financiamiento externo. A su vez, las fluctuaciones en el corto plazo respecto del equilibrio se estiman a partir de un mecanismo de corrección de errores. El período objeto de estudio es el comprendido entre 1975 y 2013. La información estadística disponible para las diferentes variables incluidas en el modelo justifica su elección.

El artículo se estructura de la siguiente forma: en la sección II se describen el marco teórico y los principales trabajos empíricos sobre modelos de crecimiento restringido por la balanza de pagos, tanto a nivel general como para el caso de Cuba. En la sección III se evalúa la correlación existente entre el PIB cubano y los flujos de comercio exterior, y se analizan los principales escenarios y políticas que pueden incidir, favorable o desfavorablemente, en el equilibrio de la balanza de pagos de Cuba y en su crecimiento económico. En la sección IV se estima un modelo de crecimiento restringido por la balanza de pagos para Cuba y se confirma la importancia que han tenido el acceso a capitales extranjeros y la firma de acuerdos preferenciales en el aligeramiento de la restricción externa cubana. Por último, en la sección $\vee$ se presentan las conclusiones y recomendaciones de política económica.

\section{Marco teórico y evidencia empírica anterior}

La identificación de los determinantes del crecimiento económico y de las diferencias de renta entre los países ha sido uno de los temas más recurrentes en la literatura económica desde los orígenes de la economía como ciencia. Autores clásicos como Adam Smith, Thomas Malthus, John Stuart Mill, David Ricardo o Karl Marx plasmaron en sus trabajos la preocupación por el crecimiento de la economía.

A partir de los resultados sobre el equilibrio estático de John Maynard Keynes, los economistas R. Harrod y D. Domar situaron en el largo plazo el análisis sobre la inestabilidad del capitalismo e inspiraron posteriores estudios sobre crecimiento equilibrado con pleno empleo, que darían forma a las teorías modernas de crecimiento económico.

Más tarde, algunos economistas cuestionaron el carácter exógeno de los factores productivos y recuperaron el papel de la demanda en la explicación del crecimiento económico. Entre los argumentos utilizados se encontraban la flexibilidad del factor trabajo para adaptarse a los ciclos económicos, la 
naturaleza de bien producido del factor capital o el crecimiento sostenido de ambos factores a lo largo de la historia. Esta línea de pensamiento, cuyo origen y fundamento teórico puede encontrarse en los trabajos de Kaldor y Thirlwall, explicaba el crecimiento a partir de la expansión de la demanda agregada y, más concretamente, por ser el único componente autónomo de esta, de las exportaciones.

Thirlwall (1979) parte del multiplicador del comercio exterior de Harrod para acabar concluyendo que, en el largo plazo, las tasas de crecimiento de las economías se encuentran restringidas por la disponibilidad de divisas, determinadas, a su vez, por las exportaciones y la elasticidad renta de la demanda de importaciones. La idea básica es que ningún país puede crecer más rápido que la tasa consistente con el equilibrio de su balanza de pagos, al no poder mantener déficits sostenidos en el tiempo.

Entre los aspectos más cuestionados de los modelos de crecimiento restringido por la balanza de pagos estuvieron el supuesto de estabilidad de precios o el hecho de no haber considerado el efecto del financiamiento externo en la determinación de la tasa de crecimiento de largo plazo (un flujo de divisas de especial relevancia para el caso de los países menos desarrollados). En respuesta a esta segunda crítica, Thirlwall y Hussain (1982) añadieron el financiamiento externo como variable explicativa del crecimiento en el largo plazo.

La evidencia empírica sobre modelos de crecimiento restringido por la balanza de pagos ofrece un elevado consenso sobre el cumplimiento de la Ley de Thirlwall. Su contraste para economías desarrolladas puede encontrarse, entre otros, en trabajos como los de McCombie y Thirlwall (1994), Atesoglu (1997) o Hieke (1997). Por su parte, los de Moreno-Brid (1998), Moreno-Brid y Pérez (1999), López y Cruz (2000), Holland, Vilela y Canuto (2002), Yongbok (2006) o Arevilca y Risso (2007) han sido algunos de los estudios en los que se ha contrastado la restricción externa al crecimiento de economías en desarrollo.

En los estudios empíricos de Mendoza y Robert (2000), Moreno-Brid (2000), Cribeiro y Triana (2005), Alonso y Sánchez (2005), Vidal y Fundora (2008) y Fugarolas, Matesans y Mañalich (2009) se ha abordado esta cuestión y se ha confirmado la validez de los modelos de crecimiento restringido por la balanza de pagos para el caso de Cuba. La elasticidad ingreso de la demanda de importaciones estimada en la literatura permite inferir que, en el largo plazo, el crecimiento de un 1\% del PIB de Cuba ha requerido un crecimiento de sus exportaciones en torno al $2 \%$. Otras conclusiones compartidas por la mayoría de los estudios son el signo negativo de la elasticidad precio de las importaciones y los problemas de estabilidad de los parámetros estimados.

\section{La restricción externa de la economía cubana: oportunidades y amenazas para el crecimiento en el corto y mediano plazo}

La consideración del equilibrio de la balanza de pagos como una restricción al crecimiento económico parece una hipótesis plausible para el caso de una economía abierta y en desarrollo como la cubana. La escasez de reservas de divisas y las dificultades a las que ha tenido que hacer frente el país para acceder a financiamiento internacional en las últimas décadas (como consecuencia del bloqueo económico y de la suspensión de pagos en 1986) se presentan como posibles causas de la gran sensibilidad que las importaciones cubanas han mostrado históricamente respecto del comportamiento de las exportaciones. En el gráfico 1 se muestra la evolución paralela que ambas series han mantenido durante el período 1975-2013, así como la fuerte correlación de ambas con la serie del PIB .

El coeficiente de correlación con el producto es de 0,85 para la serie de importaciones y de 0,96 para la serie de exportaciones. 


\section{Gráfico 1}

Cuba: PIB, exportaciones e importaciones de bienes y servicios, 1975-2013

(En millones de pesos a precios constantes de 1997)

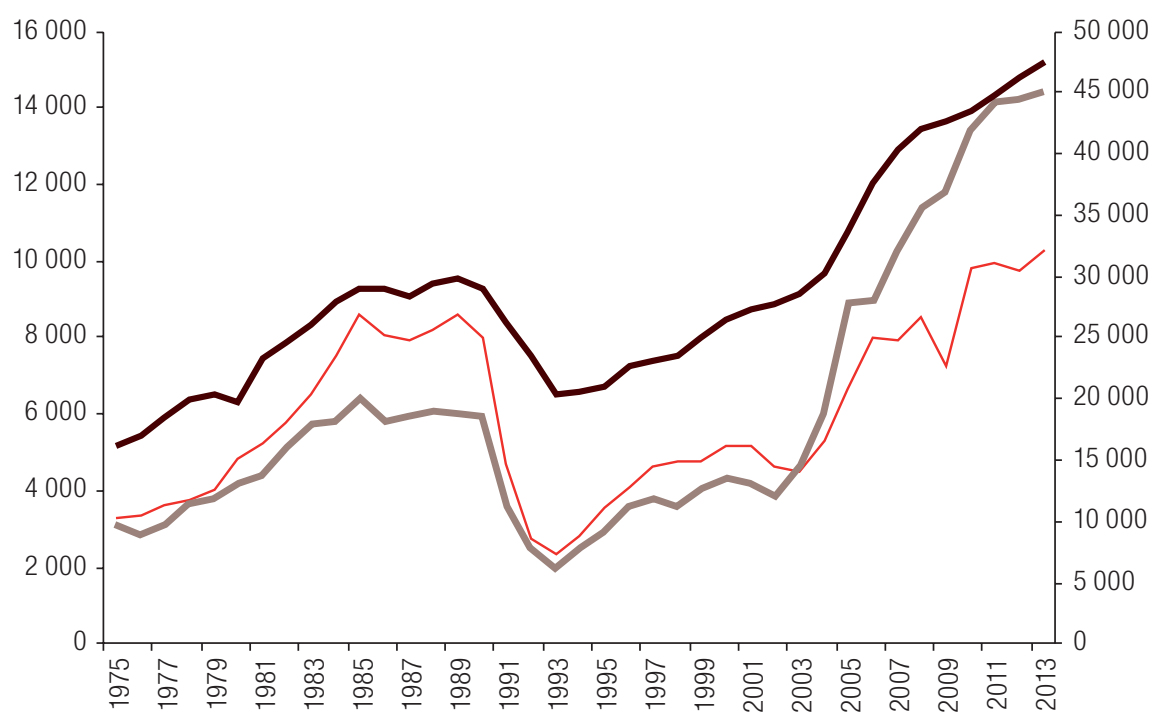

- PIB (eje derecho)

— Importaciones de bienes y servicios (eje izquierdo) — Exportaciones de bienes y servicios (eje izquierdo)

Fuente: Elaboración propia, sobre la base de datos de la Oficina Nacional de Estadística e Información (ONEI) y el Instituto Nacional de Investigaciones Económicas (INIE).

Si bien es cierto que correlación no siempre implica causalidad, el análisis de las series y de sus diferentes puntos de inflexión muestra una evidente sensibilidad del PIB cubano a los cambios en las condiciones del sector externo, lo que sugiere la causalidad en ese sentido.

De ese modo, la primera etapa de crecimiento sostenido coincide con el ingreso de Cuba al CAME, mediante lo cual se garantizaba un trato preferencial para sus exportaciones e importaciones². Un segundo punto de inflexión en la serie del PIB se corresponde con la desintegración de la URSS en 1990 y el fin de las ventajosas relaciones comerciales mantenidas con el bloque socialista. Como respuesta, a principios de los años noventa el Gobierno cubano implementó un programa de reformas que permitió la rápida reinserción del país en la economía internacional y la recuperación del PIB a partir de 1994. Esta recuperación se aceleraría a partir de 2004 como consecuencia, nuevamente, de cambios vinculados al sector externo: por una parte, el aumento de los precios internacionales de productos de gran importancia para el país, como el níquel o el tabaco (Chuairey, 2008, pág. 77), y, por la otra, los acuerdos comerciales firmados en el marco de la Alianza Bolivariana para los Pueblos de Nuestra América (ALBA). En virtud de dicho acuerdo, Cuba exportaría servicios profesionales e importaría petróleo venezolano en condiciones preferenciales. El deterioro de los términos de intercambio ${ }^{3}$, la propagación de la crisis económica internacional, la amortización de deuda cubana y la crisis de la República Bolivariana de Venezuela han vuelto a contraer las importaciones cubanas entre 2010 y 2014, reduciendo el crecimiento promedio de ese período a un exiguo 0,81\% anual ${ }^{4}$.

2 Los términos de intercambio mejoraron un 90\% entre 1973 y 1975 (ONEl, varios años).

3 Los términos de intercambio empeoraron un 38\% en 2008 (ONEl, varios años).

4 Según la Asamblea Nacional del Poder Popular cubana, en 2016 se planificó pagar alrededor de 5.299 millones de dólares en concepto de deuda externa. Véase [en línea] http://www.cubadebate.cu/opinion/2017/01/18/la-economia-cubana-20162017-valoracion-preliminar-ii/\#.WTBBN8YIHIU. 
La aparente sensibilidad del PIB cubano a la evolución de la balanza de pagos invita a revisar los factores que, en el actual contexto de incertidumbre, pueden alterar los flujos de divisas recibidos por la economía cubana durante los próximos años.

\section{Acuerdos y medidas aprobadas por el Gobierno de Obama y no revertidas por Donald Trump}

La llegada del presidente Barack Obama a la Casa Blanca supuso la flexibilización de algunos aspectos del bloqueo a Cuba y desde abril de 2009 se relajaron las restricciones para viajar y enviar remesas al país vecino ${ }^{5}$. El 17 de diciembre de 2014, durante su segundo mandato, el diferendo cubanoestadounidense viviría un día histórico: los presidentes Raúl Castro y Barack Obama anunciaban al mundo el inicio de un proceso con el que se pretendía normalizar las relaciones entre ambos países. En el ámbito político, se acordó el restablecimiento de las relaciones diplomáticas, la reapertura de las respectivas embajadas, la retirada de Cuba de la lista de países patrocinadores del terrorismo, la liberación de presos de uno y otro lado, y la eliminación de la política migratoria especial para los cubanos que lleguen a territorio estadounidense (comúnmente conocida como política de "pies secos, pies mojados"). En la esfera económica, el Gobierno de Obama impulsó medidas como la flexibilización de las condiciones para viajar a Cuba ${ }^{6}$ y para enviar remesas desde los Estados Unidos ${ }^{7}$, la aprobación de nuevas regulaciones comerciales y la autorización a los viajeros estadounidenses para abrir y mantener cuentas en Cuba, a las aerolíneas y compañías de transporte marítimo estadounidenses para viajar a Cuba sin autorización específica de la Oficina de Control de Activos Extranjeros y a las empresas estadounidenses para establecer y mantener oficinas, almacenes y otras instalaciones en Cuba en sectores como el periodístico, el agrícola, la construcción, las telecomunicaciones o las empresas de viajes ${ }^{8}$.

A continuación, se evalúa el potencial de las anteriores medidas para incrementar el volumen de divisas disponibles en Cuba. Teniendo en cuenta que los productos incluidos en los acuerdos representan un porcentaje muy pequeño del total de exportaciones cubanas de bienes, el potencial impacto del comercio de bienes es muy limitado 9 . Un segundo factor que limita considerablemente el potencial impacto de los acuerdos sobre el conjunto de exportaciones cubanas de bienes deriva del monopolio estatal del comercio exterior y de la condición de limitarlas al sector no estatal y de no favorecer al entramado empresarial del Ejército.

Muy diferente puede ser, sin embargo, el impacto de las nuevas regulaciones en los ingresos por exportaciones de servicios y, más concretamente, en los ingresos del sector turístico. A pesar de las restricciones que limitan los motivos de viaje y de los nuevos cambios anunciados por el presidente Trump (según los cuales se prohíben los viajes educativos individuales "pueblo a pueblo"),

5 Con ello se eliminaban todas las restricciones, tanto para viajar como para enviar remesas, a los residentes en los Estados Unidos con familiares cercanos en Cuba y se flexibilizaban las condiciones para el resto de los residentes en los Estados Unidos, a quienes se les permitía, a partir de ese momento, enviar dinero a Cuba (con un tope de 500 dólares por trimestre) y algunas modalidades de viaje (por motivos educativos, religiosos o "pueblo a pueblo").

6 Se autorizaron 12 motivos por los que los ciudadanos estadounidenses sin lazos familiares en la isla podrían viajar a Cuba y gastar sin límite alguno durante su estancia.

7 En septiembre de 2015 se eliminaron los límites a las remesas enviadas a Cuba en concepto de donativo o que llevaran consigo los viajeros autorizados.

8 Con el objeto de fomentar la actividad privada, el Gobierno de Obama autorizó las exportaciones a Cuba de materiales de construcción, herramientas, equipos agrícolas y productos tecnológicos vinculados a las telecomunicaciones. Asimismo, el sector no estatal cubano pasaría a poder exportar café, productos textiles y aplicaciones para móviles a empresas estadounidenses que, a su vez, podrían contratar a trabajadores cubanos.

9 En 2015, las exportaciones de café, té, cacao, especias y sus preparados apenas representaron el 0,25\% del total de exportaciones de mercancías cubanas. Las de hilados, tejidos y artículos confeccionados con fibras textiles, en tanto, solo alcanzaron el 0,01\% (ONEl, 2016). 
las nuevas regulaciones abren la posibilidad de viajar a Cuba al conjunto de estadounidenses, lo que aumenta significativamente el mercado potencial del sector turístico cubano. La relevancia de las nuevas regulaciones sobre viajes a Cuba se explica sobre la base de tres factores: la importancia del sector turístico para la economía cubana, la cercanía y el tamaño (demográfico y económico) del mercado estadounidense, y el interés que despierta viajar a un destino prohibido durante más de 55 años y con un sistema político y económico diferente ${ }^{10}$. En algunas de las proyecciones realizadas antes del cambio de Gobierno, como la de la consultora Boston Consulting Group, se estimó que el número de turistas estadounidenses que habrían visitado Cuba para 2015 superaría los dos millones anuales; un potencial que parecía avalado por el ritmo de crecimiento de los últimos años, pero que puede verse, en parte, limitado por las regulaciones del nuevo Gobierno estadounidense ${ }^{11}$.

Uno de los ámbitos en los que las nuevas regulaciones han generado resultados más visibles es, sin duda, el de las remesas. Los cambios regulatorios introducidos en 2009 por el Gobierno de Obama han permitido que las remesas a Cuba experimentaran el mayor crecimiento de toda América Latina entre 2008 y 2015, al pasar de 1.447 millones de dólares en 2008 a 3.355 millones de dólares en 2015 (Morales, 2016). La nueva flexibilización acordada en septiembre de 2015, y que no ha sido modificada por el Gobierno de Trump, hace prever que dicho crecimiento continuará en los próximos años, sobre todo si se tiene en cuenta que el volumen total de remesas recibido por Cuba sigue siendo inferior al que llega a otros países de la región con características similares.

\section{Vuelta a los mercados internacionales de capitales}

El acercamiento impulsado por Obama generó nuevas oportunidades en una esfera de especial relevancia para el crecimiento y desarrollo de un país, como el financiamiento de mediano y largo plazo a un costo razonable. El acceso de Cuba a los mercados de capitales se ha visto enormemente limitado en las últimas décadas por dos factores: el bloqueo estadounidense y el impago de la deuda en 1986. Si bien los acuerdos entre Cuba y los Estados Unidos no incluyeron el fin de las sanciones a entidades financieras, han permitido a Cuba financiar las importaciones no agrícolas y mejorar sus expectativas de crecimiento en el mediano y largo plazo (y, con ello, la confianza de los mercados) y el nivel de apoyo de la comunidad internacional al proceso de reformas ratificado en el $\mathrm{VI}$ y el VII Congreso del Partido Comunista de Cuba (PCC) $)^{12}$.

Un segundo factor clave en la mejora de la confianza de los mercados ha sido la prioridad otorgada por el Gobierno cubano al cumplimiento de los compromisos de pago acordados en el proceso de reestructuración de deuda de los últimos años. En virtud de dicha reestructuración, 14 de los 20 miembros del Club de París condonaron cerca del 80\% de la deuda cubana y extendieron el vencimiento de los pagos hasta los 18 años $^{13}$. Como resultado, la deuda externa cubana se ha reducido considerablemente en los últimos años al pasar de 35.000 millones de dólares en 2001 a 15.000 millones de dólares en 2016. Con ello, el Gobierno de Cuba ha comenzado a sentar las bases de la reinserción de la economía en los circuitos financieros internacionales. Si bien los resultados de la nueva estrategia serán lentos y graduales, la mejora de la confianza en Cuba ya ha ofrecido los primeros resultados, como queda de manifiesto en la revisión al alza de la calificación crediticia del país por parte de la agencia Moody's en 2015, en los importantes acuerdos financieros firmados en

\footnotetext{
${ }^{10}$ Con unos ingresos de 2.800 millones de dólares en 2015, el turismo representa la segunda fuente de divisas para Cuba, solo por detrás de la exportación de servicios profesionales (ONEI, 2016).

${ }^{11}$ El número de turistas estadounidenses que visitaron Cuba en 2016 triplicó la cifra registrada en 2014 (Fitzgerald, 2017).

12 Según informaba la agencia EFE en enero de 2017, entre diciembre de 2014 y enero de 2016, "la administración Obama ha multado a 11 entidades (7 estadounidenses y 4 extranjeras) por valor de unos 2.843 millones de dólares" (véase [en línea] https://www.efe.com/efe/espana/mundo/cuba-denuncia-multas-de-eeuu-a-ong-y-banco-canadiense-por-violar-el-embargo /10001-3150572).

${ }^{13}$ El importe condonado ascendió a 8.500 millones de dólares, de un total de 11.000 millones de dólares.
} 
los últimos años con Arabia Saudita, China y la Federación de Rusia o en el ingreso como socio al Banco Centroamericano de Integración Económica (BCIE) en abril de $2017^{14}$.

\section{Expansión de la inversión extranjera}

Si bien en la literatura en que se ha abordado el efecto de la IED en el crecimiento se ofrecen conclusiones dispares, en el caso de Cuba parece existir un consenso generalizado acerca del efecto positivo que esta inversión ha tenido en la economía desde su autorización en 1994 (Pérez, 1999).

Además de constituir una importante vía para acceder a nuevos capitales, la IED recibida por Cuba ha permitido el acceso a nuevas tecnologías y a mercados internacionales en sectores clave de su economía (como el turismo, la minería, la energía o las telecomunicaciones). El Gobierno cubano también parece compartir este balance positivo al identificar la promoción de la IED como un elemento fundamental del proceso de actualización del modelo, aprobar una nueva ley de IED en marzo de 2014 (mediante la cual se autorizaba la inversión extranjera en todos los sectores menos en educación, salud y defensa, y se ofrecían mayores beneficios fiscales y protección al capital foráneo) o publicar anualmente, desde 2014, una cartera de oportunidades de inversión de más de 300 proyectos con los que se busca atraer capitales extranjeros ${ }^{15}$. El vicepresidente Marino Murillo cifraba en mayo de 2014 un objetivo de entre 2.000 millones de dólares y 2.500 millones de dólares anuales de IED, muy por encima de las cantidades recibidas por Cuba desde la autorización de la IED en 1994, y que adelanta el notable crecimiento que es muy probable que esta partida experimente en los próximos años ${ }^{16}$.

A los atractivos naturales de la isla se añaden otras fortalezas, como el elevado nivel de seguridad, la estabilidad política y social, y una población altamente cualificada, que sitúan la capacidad de absorción de inversión extranjera lejos de los niveles actuales.

Sin duda, los esfuerzos para atraer capital extranjero han encontrado en las nuevas relaciones con los Estados Unidos y en los acuerdos de reestructuración de deuda dos magníficos aliados. Respecto de las inversiones procedentes de los Estados Unidos, y aunque siguen vigentes grandes limitaciones, las nuevas regulaciones han comenzado a dar sus frutos, como muestran las inversiones (autorizadas por el Departamento del Tesoro de los Estados Unidos) anunciadas por Cleber LLC o Starwood Hotels and Resorts Worldwide, o el inicio de la actividad de American Express o MasterCard en Cuba. Otra muestra del interés que ha despertado el nuevo escenario en las empresas estadounidenses puede encontrarse en las cerca de 500 autorizaciones para invertir en Cuba (por un importe global de 4.300 millones de dólares), emitidas en 2015 por el Departamento de Comercio de los Estados Unidos ${ }^{17}$. No obstante, el mayor potencial de crecimiento de la IED en el país se encuentra fuera de las fronteras estadounidenses por el mayor interés hacia Cuba que el proceso de normalización ha despertado en gran parte de la comunidad internacional tras el acercamiento de los Estados Unidos y la mejora en las expectativas de negocios - vinculadas principalmente al sector turístico- que se han abierto con el nuevo escenario ${ }^{18}$.

\footnotetext{
${ }^{14}$ En su informe de evaluación del riesgo de diciembre de 2015, la agencia Moody's elevaba las expectativas sobre Cuba de estables a positivas.

${ }^{15}$ La identificación de la promoción de la IED como un elemento fundamental del proceso de actualización del modelo queda recogida en los lineamientos 78 y 79 aprobados en el VII Congreso del PCC.

${ }^{16}$ Entre 1994 y 2013 se estima un flujo anual de IED de 328 millones de dólares.

17 Véase [en línea] https://noticias.infocif.es/noticia/cuba-la-espera-de-la-inversion-estadounidense.

${ }^{18}$ El interés que ha despertado el proceso de normalización queda demostrado en el Acuerdo de Diálogo Político y Cooperación entre Cuba y la Unión Europea, firmado el 12 de diciembre de 2016, y mediante el cual se ponía fin a la posición común.
} 


\section{Relanzamiento del proceso de reformas}

Otra importante oportunidad para dinamizar el crecimiento económico cubano y mejorar el equilibrio de la balanza de pagos podría venir del impulso al proceso de reformas. El crecimiento de las desigualdades y el incumplimiento de las expectativas generadas han causado un creciente malestar en parte de la población, que ha aumentado las resistencias internas dentro del partido y prácticamente ha detenido el proceso de implementación. A pesar de ello, el programa de reformas ratificado en el VII Congreso del PCC sigue presentando un notable potencial para aumentar los niveles de eficiencia de la economía cubana.

A tenor de los resultados cosechados hasta el momento, se antoja necesario diseñar un programa integral de reformas, compatible con un sistema socialista, que plantee en forma simultánea las medidas que sean complementarias y las secuencie en función de las condiciones necesarias para su eficaz implementación y de los resultados esperados. Entre las principales reformas aprobadas en el VI Congreso del PCC, que seis años más tarde aún siguen pendientes de aplicación, se encuentra la unificación monetaria y cambiaria. Esta es una medida central que, sin embargo, ha sido postergada principalmente por dos motivos: el impacto negativo que podría tener en los precios y en el poder adquisitivo de los salarios, y el elevado porcentaje de empresas estatales que registrarían pérdidas como consecuencia del encarecimiento de las importaciones. Una posible opción para limitar las presiones inflacionarias y conceder un mayor margen para la reestructuración empresarial y laboral podría ser una devaluación secuencial, en la que cada una de las múltiples devaluaciones parciales fuera precedida de mejoras en la productividad. Esto permitiría visibilizar el nivel de eficiencia real de las empresas y mejorar la competitividad de las exportaciones cubanas y el equilibrio en la balanza de pagos.

Entre las principales amenazas a las que se enfrenta el crecimiento económico cubano en los próximos años destacan las siguientes:

\section{a) El endurecimiento del bloqueo por parte del Gobierno de Donald Trump}

A pesar de que hasta el momento los cambios anunciados no afectan a la mayoría de los acuerdos impulsados por el Gobierno de Obama, es evidente que la actitud de Trump hacia Cuba ha enfriado las expectativas generadas por el proceso de acercamiento. Por lo tanto, en la nueva coyuntura no se descartaría un mayor endurecimiento de las condiciones que rigen las relaciones entre ambos países. El sustento principal de dicha hipótesis es que el nuevo presidente está haciendo de la reversión de las políticas de Obama su principal política. Este escenario se haría aún más factible en el caso de que el fin del chavismo en la República Bolivariana de Venezuela provocara una fuerte crisis económica en Cuba, que pudiera verse como una oportunidad para desestabilizar política y socialmente al país vecino.

No obstante, existen múltiples razones para pensar que el camino señalado por Obama en diciembre de 2014 no será desandado por completo. Entre las más relevantes cabe subrayar la valoración positiva del acercamiento a Cuba por parte de la mayoría de los votantes, tanto de Florida como del conjunto de los Estados Unidos ${ }^{19}$, los importantes intereses de empresas estadounidenses en juego (como los de los sectores agropecuario, turístico o de las telecomunicaciones), las continuas

\footnotetext{
${ }^{19}$ Según la encuesta realizada en 2016 por la Florida International University, el 64\% de los cubano-estadounidenses de Miami apoya las políticas de acercamiento impulsadas por Obama. Otra encuesta, publicada en febrero de 2014 por el Atlantic Council, situaba el nivel de apoyo al deshielo en un 64\% en el conjunto de los Estados Unidos y en un 67\% en Miami.
} 
visitas a Cuba de gobernadores y legisladores del Partido Republicano, la firma de importantes acuerdos portuarios o la demostrada ineficacia del bloqueo ${ }^{20}$.

\section{b) El cambio de Gobierno en la República Bolivariana de Venezuela}

Las relaciones de Cuba con la República Bolivariana de Venezuela han ido estrechándose desde 2000 hasta hacer de esta última el principal inversionista extranjero y socio comercial de la mayor de las Antillas ${ }^{21}$. Este nivel de dependencia se ha hecho aún más evidente con la grave crisis política y económica por la que atraviesa la República Bolivariana de Venezuela y ha supuesto una reducción del 51\% en el intercambio comercial entre ambos países entre 2012 y 2015 (ONEl, 2017), el recorte en el suministro de petróleo venezolano (de 105.000 barriles diarios exportados hasta septiembre de 2014 a 55.000 barriles diarios suministrados desde entonces), la contracción de las importaciones de servicios médicos cubanos y la ausencia de inversiones venezolanas en Cuba (Mesa-Lago, 2015, pág. 3). Como resultado, en 2016 el PIB cubano se alejaba del crecimiento planificado del $2 \%$ para retroceder un 0,9\%, en la que ha sido la primera contracción del producto desde 1993.

\section{Estimación de un modelo de crecimiento restringido por la balanza de pagos para el caso cubano}

La idea principal en la que se basa el modelo propuesto por Thirlwall (1979) es que los desequilibrios en la cuenta corriente no pueden mantenerse indefinidamente en el tiempo, por lo que es necesario que en el largo plazo el crecimiento de los países sea compatible con el equilibrio de su balanza de pagos. En su análisis, el autor sitúa las exportaciones y la elasticidad ingreso de la demanda de importaciones como las variables fundamentales en la determinación del crecimiento económico de largo plazo.

Una derivación lógica del modelo es el hecho de que todas las economías del mundo no pueden estar restringidas por la balanza de pagos al mismo tiempo, si bien bastaría con que un país o un grupo de países no estuviesen restringidos para que el resto sí lo estuviese.

El análisis parte de la identidad que define el equilibrio de la balanza de pagos:

$$
P_{t}^{d} X_{t}+E_{t} F_{t}=P_{t}^{f} M_{t}
$$

Donde $X_{t}$ representa las exportaciones reales, $P_{t}^{d}$ el precio de las exportaciones en moneda nacional, $F_{t}$ el flujo de capital medido en moneda extranjera, $M_{t}$ las importaciones reales, $P_{t}^{f}$ el precio de las importaciones en moneda extranjera y $E_{t}$ el tipo de cambio nominal expresado en unidades de moneda nacional por cada unidad de moneda extranjera.

En el modelo planteado, la economía tiene dos fuentes de divisas: las exportaciones y el financiamiento externo. La participación relativa de las primeras en el volumen total de divisas viene dada por la siguiente expresión:

\footnotetext{
${ }^{20}$ Los puertos de Lake Charles, Luisiana, Nueva Orleans, Alabama y Virginia han firmado acuerdos con Cuba, incluso durante la actual legislatura.

${ }^{21}$ Entre 2010 y 2015, el 40\% del total de los intercambios comerciales de Cuba fueron con la República Bolivariana de Venezuela (ONEl, 2017), país que satisfizo más de la mitad de las necesidades cubanas de combustible e importó la mayoría de sus servicios médicos (Quiñones y Mañalich, 2010, pág. 11).
} 


$$
\theta=\frac{P_{t}^{d} X_{t}}{P_{t}^{d} X_{t}+E_{t} F_{t}}
$$

El análisis asume demandas de exportación y de importación, expresadas en función de los niveles de renta y de precios, y con elasticidades constantes.

$$
\begin{gathered}
X_{t}=\left(\frac{P_{t}^{d}}{E_{t} P_{t}^{f}}\right)^{\Psi} Z_{t}^{n} \quad \begin{array}{l}
\psi, p>0 \\
\eta, f>0
\end{array} \\
M_{t}=\left(\frac{E_{t} P_{t}^{f}}{P_{t}^{d}}\right)^{\phi} Y_{t}^{\pi} \quad \begin{array}{l}
\phi, p>0 \\
\end{array}, f>0
\end{gathered}
$$

Donde $Z_{t}$ representa la renta mundial, $Y_{t}$ la renta nacional, $\Psi$ y $\eta$ las elasticidades precio y renta de la demanda de exportaciones, y $\phi$ y $\pi$ las elasticidades precio y renta de la demanda de importaciones.

Si se toman logaritmos, las ecuaciones (1), (3) y (4) pueden expresarse en tasas de crecimiento:

$$
\begin{gathered}
\theta\left(p_{t}^{d}+x_{t}\right)+(1-\theta)\left(e_{t}+f_{t}\right)=e_{t}+p_{t}^{f}+m_{t} \\
m_{t}=\phi\left(p_{t}^{f}-e_{t}-p_{t}^{d}\right)+\pi y_{t} \\
x_{t}=\psi\left(p_{t}^{d}+e_{t}-p_{t}^{f}\right)+\eta z_{t}
\end{gathered}
$$

La combinación de las ecuaciones (5), (6) y (7) permite expresar la tasa de crecimiento compatible con la balanza de pagos como sigue:

$$
y_{t}=\frac{\theta \eta z_{t}+(\theta \psi+\phi+1)\left(p_{t}^{d}-e_{t}-p_{t}^{f}\right)+(1-\theta)\left(e_{t}+f_{t}-p_{t}^{d}\right)}{\pi}
$$

Una expresión que puede simplificarse si se asume que en el largo plazo se cumple con la paridad del poder adquisitivo $\left(p_{t}^{d}-e_{t}-p_{t}^{f}=0\right)$ y el tipo de cambio se mantiene estable $\left(e_{t}=0\right)$.

$$
y_{t}=\frac{\theta \eta z_{t}+(1-\theta)\left(f_{t}-p_{t}^{d}\right)}{\pi}
$$

Thirlwall (1979) considera además que los flujos de capital se mantienen estables en el tiempo $\left(f_{t}-p_{t}^{d}\right)$ o representan un porcentaje pequeño respecto del total de divisas $(\theta=1)$, por lo que combinando las ecuaciones (7) y (8) puede derivarse la siguiente ecuación:

$$
y_{t}=\frac{x_{t}}{\pi}
$$

La ecuación (10), conocida en la literatura como Ley de Thirlwall, permite estimar la tasa de crecimiento compatible con el equilibrio en la balanza de pagos a partir del crecimiento de las exportaciones y de la elasticidad ingreso de las importaciones. 
Los datos utilizados en el análisis econométrico son series anuales a nivel agregado para el período 1975-2013.

La serie del PIB se incluye a precios constantes de mercado (con base en 1997) y se obtiene a partir de ONEI (varios años). El cambio metodológico en la medición del PIB cubano hace recomendable la corrección de la serie original para el período 2007-2013. Los servicios sociales y personales que, hasta ese momento, habían sido valorados en virtud de sus costos, pasaron a calcularse a precios estimados de mercado. Como resultado, el producto de este sector creció en 2004 más de un 84\% (pasó de representar el 11,6\% del PIB en 2003 al 31,3\% del PIB en 2004) y desde entonces infló las tasas de crecimiento a nivel agregado22. A partir de ese año, el producto se recalcula aplicando la tasa de crecimiento de la esfera productiva (no afectada por el cambio de metodología) al dato del año anterior ${ }^{23}$.

Los datos de exportaciones de bienes y servicios se obtienen a partir del detalle de la balanza de pagos incluido en los anuarios estadísticos de Cuba y de información facilitada por el Instituto Nacional de Investigaciones Económicas (INIE).

En gran parte de la literatura sobre crecimiento restringido por la balanza de pagos, la variable de financiamiento externo es aproximada mediante el déficit en cuenta corriente. El interés por captar el efecto que las remesas y la IED han podido tener en la economía cubana justifica que en la aproximación del financiamiento externo que se plantea se diferencie entre los principales flujos externos de esta economía: créditos procedentes del exterior, remesas e IED. Los créditos externos se aproximan mediante la diferencia entre el déficit comercial (ONEl, varios años) y los flujos de IED y de remesas. Esto al considerar que en una economía sin reservas de divisas, como la cubana, la mayor parte del déficit no financiado mediante IED ni remesas deberá financiarse por medio de créditos externos ${ }^{24}$.

Dado que los anuarios estadísticos solo cuentan con datos oficiales sobre el flujo de IED de Cuba para el período 1994-2001, se hace necesario completar la serie a partir de las estimaciones de la Economist Intelligence Unit (s/f).

Del mismo modo, los datos oficiales de remesas se encuentran disponibles únicamente para el período comprendido entre 1994 y 2004 (ONEl, varios años), por lo que es necesario completar la serie a partir de los datos del Havana Consulting Group para el período 2005-2013. Ambas series se expresan en precios corrientes. Para los términos de intercambio se utilizan los datos facilitados por la ONEI y el INIE.

En el gráfico 2 se presenta la evolución de las series incluidas en el análisis.

22 Para 2004, 2005, 2007 y 2008, las tasas de crecimiento del conjunto de la economía eran más del doble que las observadas en la esfera productiva (ONEI, varios años).

${ }^{23}$ Se asumen las tasas de crecimiento y la definición de sector productivo propuesta por la ONEl, que incluye al sector agropecuario, industria, minería y energía, turismo, construcción y transporte y comunicaciones.

24 Siguiendo a Vidal y Fundora (2008), a toda la serie se suma dos veces el valor mínimo en valor absoluto para no tener datos negativos en los años en que el déficit en cuenta corriente fue inferior al volumen de IED, con el objeto de expresar las variables en logaritmos y obtener sus elasticidades. 


\section{Gráfico 2}

Evolución de las series, 1975-2013

(En logaritmo de los datos)

A. LCRE

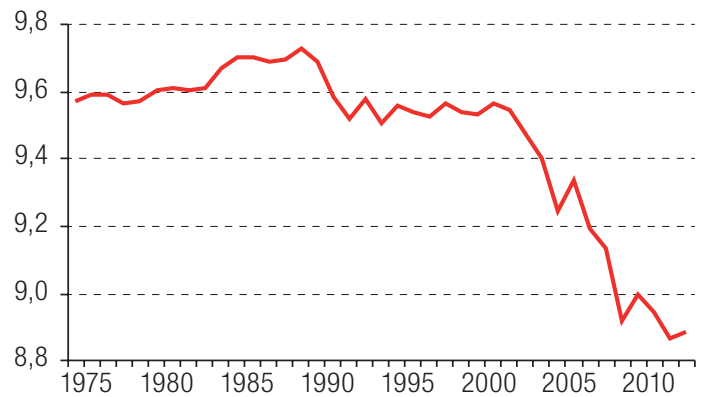

C. LREM

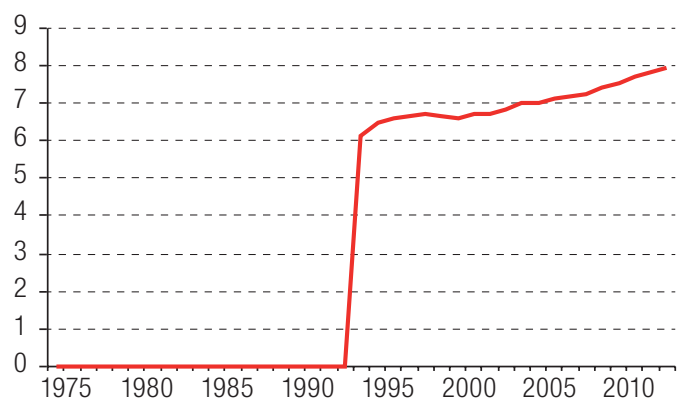

E. LY

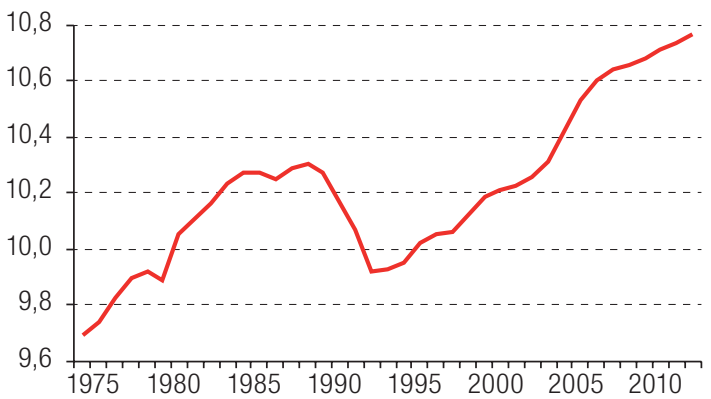

B. LIED

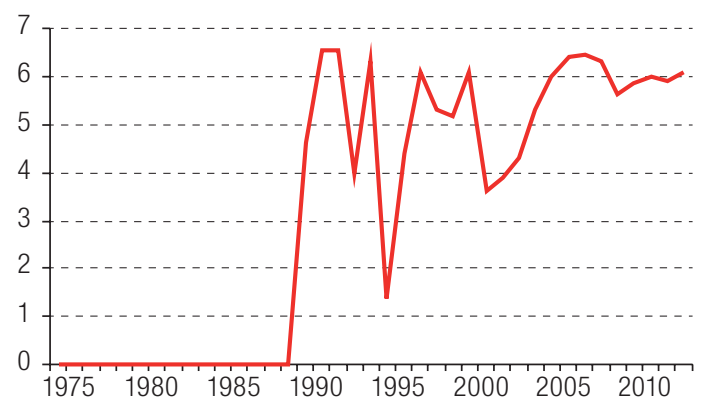

D. LTIT

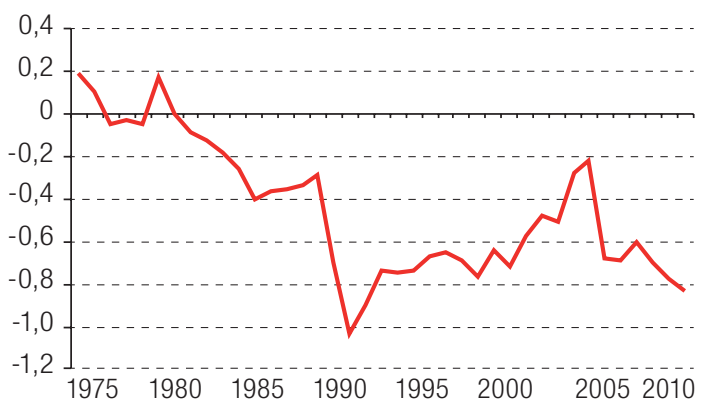

F. LXBS

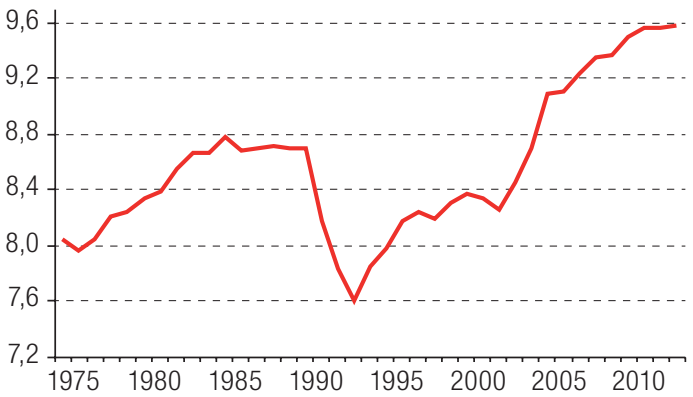

Fuente: Elaboración propia.

Nota: CRE: financiamiento externo vía créditos; IED: inversión extranjera directa; REM: remesas; TIT: términos de intercambio; Y: PIB; XBS: exportaciones de bienes y servicios. Las series LIED y LREM se inician en 1990 y 1994 porque hasta entonces no estaban autorizadas ni las inversiones extranjeras ni las remesas.

La estacionariedad de las series se analiza a partir de las pruebas de Dickey-Fuller aumentadas. Sus resultados se muestran en el cuadro 1. 


\section{Cuadro 1}

Prueba de estacionariedad de las series, 1975-2013a

(Resultados estadísticos con el logaritmo de los datos)

\begin{tabular}{lccc}
\hline & Estadístico-t & Constante y tendencia & Rezagos \\
\hline$Y$ & $-1,846$ & Sí & 1 \\
\hline XBS & 0,962 & No & 1 \\
\hline REM & 0,403 & No & 0 \\
\hline IED & $-1,723$ & Constante & 0 \\
\hline TIT & $-1,933$ & Constante & 0 \\
\hline CRE & $-1,702$ & No & 0 \\
\hline$\Delta Y$ & $-2,927^{*}$ & No & 0 \\
\hline$\Delta$ XBS & $-3,561^{*}$ & No & 0 \\
\hline$\Delta$ REM & $-5,673^{*}$ & No & 0 \\
\hline$\Delta$ IED & $-7,790^{*}$ & No & 1 \\
\hline$\Delta$ TIT & $-5,527^{*}$ & No & 0 \\
\hline$\Delta$ CRE & $-6,718^{*}$ & Constante & 0 \\
\hline Resid & $-6,672^{* *}$ & No & 0 \\
\hline Fuente: & & & 0
\end{tabular}

Fuente: Elaboración propia.

Nota: Y: PIB; XBS: exportaciones de bienes y servicios; REM: remesas; IED: inversión extranjera directa; TIT: términos de intercambio; CRE: financiamiento externo vía créditos.

a Prueba de Dickey-Fuller aumentada.

b Residuos estimados de la ecuación 12.

Estacionaria al 1\%.

* Los valores calculados del estadístico t (tau) se comparan con los valores críticos tabulados en Engle y Yoo (1987), dado que, en este caso, los valores críticos de Dickey-Fuller o de MacKinnon (1996) no derivan de la realización directa de un proceso estocástico subyacente.

Los resultados de las pruebas de Dickey-Fuller aumentadas muestran que todas las series utilizadas en el análisis econométrico son no estacionarias con orden de integración 1, l(1), lo que permite analizar la posible relación de cointegración existente entre ellas.

Para eso se estima alternativamente la transformación logarítmica de las ecuaciones (8) y (10), con el objeto de identificar cuál de las dos especificaciones aproxima mejor el crecimiento económico cubano de las últimas décadas. Además, se incluye en el modelo una variable ficticia que adopta el valor 1 para los años en que Cuba ha mantenido acuerdos comerciales preferentes y el valor 0 para el resto, con el propósito de captar los beneficios derivados de dichos acuerdos, no recogidos en las estadísticas oficiales ${ }^{25}$.

$$
\log Y_{t}=\alpha_{1}+\alpha_{2} \log X B S_{t}+e_{t}
$$

$$
\log Y_{t}=\beta_{0}+\beta_{1} \log X B S_{t}+\beta_{2} \log I E D_{t}+\beta_{3} \log R E M_{t}+\beta_{4} \log T I T_{t}+\beta_{5} \log C R E_{t}+A P_{t}+e_{t}
$$

Donde $e_{t}$ es el término de error aleatorio ruido blanco y los parámetros $\beta$ representan las elasticidades de largo plazo de las respectivas variables explicativas.

Los resultados de la estimación por mínimos cuadrados ordinarios (MCO) de las ecuaciones (11) y (12) se presentan en el cuadro 2.

\footnotetext{
${ }^{25}$ Cuba mantuvo acuerdos comerciales preferentes entre 1975 y 1989 con la URSS y entre 2001 y 2013 con la República Bolivariana de Venezuela.
} 


\section{Cuadro 2}

Cuba: estimación por mínimos cuadrados ordinarios (MCO) de las ecuaciones (11) y (12)a (Resultados estadísticos con el logaritmo de los datos)

\begin{tabular}{|c|c|c|c|c|}
\hline \multirow{2}{*}{$\mathrm{PIB}_{t}$} & \multicolumn{2}{|c|}{ Ecuación (11) } & \multicolumn{2}{|c|}{ Ecuación (12) } \\
\hline & Coeficiente & t-estadístico & Coeficiente & t-estadístico \\
\hline Constante & $5,920^{b}$ & 19,737 & $3,419^{b}$ & 5,893 \\
\hline XBS & $0,501^{b}$ & 14,993 & $0,496^{b}$ & 29,279 \\
\hline IED & - & - & $0,025^{b}$ & 3,274 \\
\hline CRE & - & - & $0,239^{b}$ & 4,938 \\
\hline REM & - & - & $-0,007^{c}$ & $-2,425$ \\
\hline TIT & & & $-0,324^{b}$ & $-7,186$ \\
\hline $\mathrm{AP}$ & & & $0,114^{b}$ & 4,374 \\
\hline $\mathrm{R}^{2}$ ajustado & 0,865 & & 0,980 & \\
\hline Akaike (Schwarz) & $-1,598$ & $(-1,513)$ & $-3,424$ & $(-3,126)$ \\
\hline Durbin-Watson & 0,322 & & 2,020 & \\
\hline
\end{tabular}

Fuente: Elaboración propia.

Nota: XBS: exportaciones de bienes y servicios; IED: inversión extranjera directa; CRE: financiamiento externo vía créditos; REM: remesas; TIT: términos de intercambio.

a Errores estándares corregidos por heterocedasticidad (Huber-White).

b Significativo al $1 \%$.

c Significativo al $5 \%$.

La mayor capacidad explicativa mostrada por el $\mathrm{R}^{2}$ ajustado y la mejoría en los valores de Akaike y Schwarz y en el estadístico Durbin-Watson confirman la conveniencia de utilizar, para el caso cubano, modelos de crecimiento restringido por la balanza de pagos en los que también se consideren los términos de intercambio y otros flujos de divisas diferentes a las exportaciones (como la IED, los créditos procedentes del exterior o las remesas). Ambos modelos cumplen con los supuestos de homocedasticidad y normalidad de los residuos y de ausencia de cambio estructural. Los problemas de autocorrelación detectados en los residuos estimados en la ecuación (11) desaparecen con la inclusión en la ecuación (12) del resto de flujos de divisas, los términos de intercambio y la variable ficticia (véase el anexo).

La cointegración entre las variables se analiza a partir de la metodología desarrollada en Engle y Granger (1987), según la cual puede encontrarse un vector de cointegración que cuando las variables del modelo sean integradas del mismo orden permita una combinación de estas de orden menor ${ }^{26}$. En nuestro caso, los resultados de la prueba de Dickey-Fuller aumentada incluidos en el cuadro 1 muestran que los residuos estimados son estacionarios, lo que confirma la relación de cointegración entre las variables del modelo ${ }^{27}$. El hecho de descartar que la correlación entre las variables se deba a una posible relación espuria permite interpretar los parámetros del modelo en logaritmos como las elasticidades a largo plazo. Todos los parámetros resultan significativos con un nivel de confianza del 95\%.

La elasticidad renta de las exportaciones estimada (2,02), calculada en los modelos de crecimiento restringido por la balanza de pagos como la inversa del parámetro asociado a las exportaciones $\left(1 / \beta_{2}\right)$, resulta significativa, positiva y muy próxima a los valores estimados en trabajos anteriores en los que se han aplicado modelos de crecimiento restringido por la balanza de pagos al caso cubano. Respecto de la IED, su elasticidad confirma el efecto positivo en el crecimiento de

\footnotetext{
${ }^{26}$ En el presente trabajo se opta por la metodología de cointegración desarrollada en Engle y Granger, puesto que el test de Johansen registra algunos problemas en el caso de muestras pequeñas, al generar en ese caso matrices casi singulares (Matesanz, Fugarolas y Candaudap, 2007, pág. 36).

27 Para el contraste de cointegración, los valores calculados del estadístico t (tau) se comparan con los valores críticos tabulados en Engle y Yoo (1987), dado que, en este caso, los valores críticos de Dickey-Fuller o de MacKinnon (1996) no derivan de una realización directa de un proceso estocástico subyacente, sino que son el resultado de una serie construida después de estimar los parámetros del modelo.
} 
la economía cubana. De igual modo, el signo positivo del parámetro asociado a la variable CRED, conforme a lo esperado, sugiere que la rentabilidad de las inversiones cubanas financiadas con créditos externos ha sido mayor que los altos intereses cobrados por dichos préstamos. Por otra parte, la elasticidad precio negativa recogida en el signo del parámetro asociado a los términos de intercambio coincide con el obtenido en anteriores estudios del caso cubano y podría explicarse por la menor relevancia de la variable precio en los intercambios comerciales entre Cuba y el área del CAME, y por la estructura de las importaciones cubanas, con un elevado porcentaje destinado a bienes básicos y, por lo tanto, con una demanda menos sensible a la evolución de los precios. El único parámetro para el que se obtiene un resultado contrario a lo esperado es el asociado a las remesas. Si bien es cierto que la falta de información pública y homogénea sobre las remesas que recibe la isla obliga a ser cauto en la valoración de los resultados, su signo negativo podría deberse al restrictivo marco regulatorio cubano en materia de inversión, que limita enormemente la inversión productiva de las remesas y obliga a destinarlas, en su totalidad, a mejorar la cesta de consumo de sus beneficiarios o a actividades de muy bajo valor añadido y alta informalidad.

Tras la estimación de largo plazo, el cumplimiento del teorema de representación de Granger - según el cual si un vector de variables es $\mathrm{Cl}(1,1)$ existe un mecanismo de corrección de errores (MCE) válido para representar el proceso generador de datos (Intriligator, Bodkin y Hsiao, 1996)permite aplicar en nuestro análisis un modelo MCE. Al combinar variables en niveles con variables en primeras diferencias, dicho modelo permite vincular el análisis de equilibrio de largo plazo con la dinámica de ajuste de corto plazo y estimar la velocidad con que se corrigen los posibles desajustes respecto del equilibrio de largo plazo.

Para ello, se sigue la metodología en dos etapas de Engle y Granger, consistente en incluir en el modelo MCE los residuos estimados retardados un período.

$$
\begin{gathered}
\Delta \log Y_{t}=\beta_{0}+\beta_{1} \Delta \log X B S_{t}+\beta_{2} \Delta \log I E D_{t}+\beta_{3} \Delta \log C R E_{t}+\beta_{4} \Delta \log R E M_{t} \\
+\beta_{5} \Delta \log T_{I T}+\beta_{6} A P_{t}+\beta_{7} \operatorname{errorLP}_{t-1}+e_{t}
\end{gathered}
$$

Los resultados de la estimación de la ecuación (13) se muestran en el cuadro 3, donde también se incluyen los resultados obtenidos después de eliminar las variables con parámetros no significativos en la primera regresión.

Con un nivel de confianza del 95\%, el modelo cumple con los supuestos de homocedasticidad, no autocorrelación y normalidad de los residuos (véase el anexo). Por su parte, la relación de cointegración se ve confirmada con la significancia del término de corrección de errores. El parámetro de velocidad del ajuste estimado es de 0,706, lo que muestra que las desviaciones temporales del equilibrio de largo plazo se ajustan por completo en menos de dos años. Las elasticidades estimadas en el corto plazo mantienen el signo para todas las variables, si bien en el corto plazo solamente las exportaciones, los términos de intercambio y la variable ficticia son significativas. Esto sugiere que los créditos, la inversión extranjera y las remesas requieren de períodos más largos para concretar su efecto en el PIB. 
Cuadro 3

Mecanismo de corrección de errores ${ }^{a}$

(Resultados estadísticos con el logaritmo de los datos)

\begin{tabular}{|c|c|c|c|c|}
\hline \multirow{2}{*}{$\Delta \mathrm{PIB}_{t}$} & \multicolumn{2}{|c|}{ Ecuación (13) } & \multicolumn{2}{|c|}{ Ecuación (13) } \\
\hline & Coeficiente & t-estadístico & Coeficiente & t-estadístico \\
\hline Constante & $-0,006$ & $-0,689$ & - & - \\
\hline$\Delta \mathrm{XBS}$ & $0,351^{c}$ & 6,552 & $0,337^{c}$ & 5,424 \\
\hline$\Delta \mathrm{IED}$ & 0,007 & 1,937 & - & - \\
\hline$\Delta \mathrm{CRE}$ & 0,183 & 1,551 & - & - \\
\hline$\Delta$ REM & $-0,001$ & $-0,150$ & - & - \\
\hline$\Delta \mathrm{TIT}$ & $-0,204^{c}$ & $-2,722$ & $-0,176^{c}$ & $-2,738$ \\
\hline $\mathrm{AP}$ & $0,024^{c}$ & 2,304 & $0,016^{d}$ & 2,121 \\
\hline errorLP(-1) & $-0,722^{c}$ & $-2,883$ & $-0,706^{c}$ & $-3,069$ \\
\hline $\mathrm{R}^{2}$ ajustado & 0,682 & & 0,666 & \\
\hline Akaike (Schwarz) & $-3,817$ & $(-3,473)$ & $-3,852$ & $(-3,680)$ \\
\hline Durbin-Watson & 1,886 & & & 1,931 \\
\hline
\end{tabular}

Fuente: Elaboración propia.

Nota: XBS: exportaciones de bienes y servicios; IED: inversión extranjera directa; CRE: financiamiento externo vía créditos; REM: remesas; TIT: términos de intercambio.

a Errores estándares corregidos por heterocedasticidad (Huber-White).

b Estimación de la ecuación (13), excluidas las variables no significativas al 5\%.

Significativo al $1 \%$.

Significativo al $5 \%$.

\section{Conclusiones y recomendaciones de política económica}

La condición de pequeña economía abierta, sin reservas de divisas y con un escaso y oneroso acceso a los mercados internacionales de capitales explica la alta sensibilidad que muestra el crecimiento económico cubano respecto del comportamiento de su sector exterior.

El análisis de cointegración propuesto confirma dicha hipótesis y permite concluir que, si bien el impacto de los flujos de remesas en el PIB no ha sido el esperado durante el período de estudio, la IED, los créditos exteriores y los acuerdos preferenciales han tenido un efecto significativo y positivo en la determinación de las tasas de crecimiento de largo plazo de la economía cubana.

En consecuencia, el desarrollo de políticas industriales y comerciales dirigidas a reducir la dependencia importadora representa uno de los principales retos de la economía de la isla para el largo plazo. También debe tenerse muy en cuenta la restricción externa a la hora de diseñar una política económica capaz de afrontar las principales amenazas y oportunidades a las que se enfrenta el país en los próximos años. El cambio de Gobierno en la República Bolivariana de Venezuela o el endurecimiento del bloqueo por parte del Gobierno de Trump socavarían notablemente el volumen de divisas disponibles en Cuba y es muy probable que conduzcan al país a una nueva crisis económica, en un momento de mayor incertidumbre política, luego de la llegada al poder de Miguel Díaz-Canel, como sucesor de Raúl Castro. Ante ese posible escenario, parece razonable que el Gobierno cubano aproveche las oportunidades que le brinda el nuevo marco de relaciones con los Estados Unidos y reimpulse el proceso de reformas internas.

Muy probablemente, flexibilizar la restricción externa cubana también requerirá estimular la IED, continuar con el proceso de vuelta a los mercados de capitales e introducir cambios en la regulación 
que permitan la inversión productiva de las remesas. Para ello, las inversiones en el sector no estatal deberían abrirse a profesiones y actividades de mayor productividad, permitiendo aprovechar el enorme capital humano del que dispone Cuba (actualmente subempleado en actividades que apenas requieren formación). El potencial y la sostenibilidad del nuevo marco dependerán, a su vez, de la capacidad del Gobierno cubano para compatibilizar la mejora de la eficiencia y la expansión de las capacidades productivas con el respeto por los principios socialistas, priorizando formas de propiedad cooperativas e implementando una política tributaria que limite las desigualdades y permita mejorar el salario real del conjunto de la población.

\section{Bibliografía}

Alonso, J. A. y J. M. Sánchez (2005), "La competitividad desde una perspectiva macro: la restricción externa al crecimiento", Tecnología, competitividad y capacidad exportadora de la economía cubana: el desafío de los mercados globales, La Habana.

Arevilca, B. J. y A. W. Risso (2007), "Balance of payments constrained growth model: evidence for Bolivia 1953-2002”, MPRA Paper, № 5645 [en línea] https://mpra.ub.uni-muenchen.de/5645/1/MPRA_paper_ 5645.pdf.

Atesoglu, H. S. (1997), "Balance-of-payments-constrained growth model and its implications for the United States", Journal of Post Keynesian Economics, vol. 19, Nㅜ 3, Taylor \& Francis.

Chuairey, A. (2008), "La sustitución de importaciones en Cuba: un enfoque de elasticidades", tesis, La Habana, Universidad de la Habana.

Cribeiro, Y. y L. Triana (2005), "Las elasticidades en el comercio exterior cubano: dinámica de corto y largo plazo", tesis, La Habana, Facultad de Economía, Universidad de La Habana.

Economist Intelligence Unit (s/f), "Country Report Cuba" [en línea] https://store.eiu.com/product/countryreport/cuba.

Engle, R. F. y C. Granger (1987), "Co-integration and error correction: representation. Estimation and testing", Econometrica, vol. 55, № 2, Nueva York, The Econometric Society.

Engle, R. F. y B. S. Yoo (1987), "Forecasting and testing in co-integrated systems", Journal of Econometrics, vol. 35, No 1, Amsterdam, Elsevier.

Fitzgerald, M. (2017), "Taking the long view on Cuba's tourism opportunity" [en línea] https://www.bcg.com/ publications/2017/transportation-travel-tourism-winning-emerging-markets-taking-long-view-cubastourism-opportunity.aspx.

Fugarolas, A. U., D. Matesans e I. Mañalich (2009), "Empirical evidence of the balance of payments constrained growth in Cuba. The effects of commercial regimes since 1960", Versus Acadêmica, vol. 3.

Hieke, H. (1997), "Balance-of-payments-constrained growth: a reconsideration of the evidence for the U.S. economy", Journal of Post Keynesian Economics, vol. 19, № 3, Taylor \& Francis.

Holland, M., F. V. Vilela y O. Canuto (2002), "Economic Growth and the Balance of Payments Constraint in Latin America", documento presentado en el VII Encuentro Nacional de Economía Política [en línea] http://www.sep.org.br/eventos.asp?evento=s_sete.

Intriligator, M., R. Bodkin y C. Hsiao (1996), Econometric Models, Techniques and Applications, New Jersey, Prentice Hall.

López, J. y A. Cruz (2000), "Thirlwall's law and beyond: the Latin American experience", Journal of Post Keynesian Economics, vol. 22, № 3, Taylor \& Francis.

MacKinnon, J. (1996), "Numerical distribution functions for unit root and cointegration tests", Journal of Applied Econometrics, vol. 11, № 6, Wiley.

Matesanz, D., G. Fugarolas y E. Candaudap (2007), "Balanza de pagos y crecimiento económico restringido. Una comparación entre la economía argentina y la mexicana”, Revista de Economía Mundial, № 107, Huelva, Sociedad de Economía Mundial.

McCombie, J. S. L. y A. P. Thirlwall (1994), Economic Growth and the Balance-of-Payments Constraint, Nueva York, Palgrave Macmillan.

Mendoza, Y. y L. Robert (2000), "El crecimiento económico y las restricciones en el sector externo. Una aplicación al caso cubano", Cuba: Investigación Económica, año 6, №3, La Habana, Instituto Nacional de Investigaciones Económicas (INIE). 
Mesa-Lago, C. (2015), "Normalización de relaciones entre EEUU y Cuba: causas prioridades, progresos, obstáculos, efectos y peligros", Documento de Trabajo, № 6/2015, Madrid, Real Instituto Elcano.

Morales, E. (2016), "Cuba: the fastest growing remittances market in Latin America", The Havana Consulting Group and TECH [en línea] http://www.thehavanaconsultinggroup.com/en-/Articles/Article/20.

Moreno-Brid, J. C. (2000), "Crecimiento económico y escasez de divisas", La economía cubana. Reformas estructurales y desempeño en los noventa (LC/G.2020/Rev.1), Ciudad de México, Comisión Económica para América Latina y el Caribe (CEPAL)/Fondo de Cultura Económica.

- (1998), "On capital flows and the balance-of-payments constrained growth model", Journal of Post Keynesian Economics, vol. 21, № 2, Taylor \& Francis.

Moreno-Brid, J. C. y E. Pérez (1999), "Balanza de pagos y crecimiento en América Central, 1950-1996", Comercio Exterior, vol. 50, № 1, Ciudad de México, Banco Nacional de Comercio Exterior (BANCOMEXT).

ONEI (Oficina Nacional de Estadísticas e Información) (2017), Anuario Estadístico de Cuba, 2016, La Habana. (2016), Anuario Estadístico de Cuba, 2015, La Habana. (varios años), Anuario Estadístico de Cuba, La Habana.

Pérez, O. E. (1999), "La inversión extranjera directa en Cuba. Peculiaridades", Centro de Estudios de la Economía Cubana (CEEC) [en línea] https://www.nodo50.org/cubasigloXXI/economia/villanueva2_300602.htm.

Quiñones, N. e I. Mañalich (2010), "El sector externo cubano y sus retos estructurales", La Habana, Instituto Nacional de Investigaciones Económicas (INIE).

Thirlwall, A. (1979), "The balance of payments constraint as an explanation of international growth rate differences", BNL Quarterly Review, vol. 32, № 128, Banca Nazionale del Lavoro.

Thirlwall, A. P. y M. N. Hussain (1982), "The balance of payments constraint, capital flows and growth rate differences between developing countries", Oxford Economic Papers, vol. 34, N 3, Oxford, Oxford University Press.

Vidal, P. y A. Fundora (2008), "Relación comercio-crecimiento en Cuba: estimación con el filtro de Kalman", Revista de la CEPAL, № 94 (LC/G.2357-P), Santiago, Comisión Económica para América Latina y el Caribe (CEPAL).

Yongbok, J. (2006), "Balance-of-payment-constrained growth: the case of China, 1979-2002", Documento de Trabajo, № 6, Salt Lake City, Utah, Universidad de Utah. 


\section{Anexo A1}

\section{Cuadro A1.1}

Test estadísticos.

Estimación por mínimos cuadrados ordinarios (MCO)

\begin{tabular}{lrrc}
\hline Ecuación & BPa $^{a}$ & BPG $^{b}$ & Jarque Bera \\
\hline$(1)$ & 1,010 & 1,379 & 4,451 \\
\hline$(2)$ & 22,855 & 14,481 & 0,858 \\
\hline$(3)^{c}$ & 0,741 & 0,641 & 2,655 \\
\hline
\end{tabular}

Fuente: Elaboración propia.

a Test de autocorrelación de Breusch-Pagan.

b Test de heterocedasticidad de Breusch-Pagan-Godfrey.

c Estimación de la ecuación (13) después de eliminar las variables redundantes. 